ISSN: 2705-313X (PRINT); 2705-3121 (ONLINE)

Research Article

Open Access Journal available at: https://cajostssu.com and https://www.ajol.info/index.php/cajost/index

This work is licensed under a Creative Commons Attribution-NonCommercial 4.0 International License. DOI: https://dx.doi.org/10.4314/cajost.v4i1.2

\author{
Article Info \\ Received: $8^{\text {th }}$ April 2021 \\ Revised: $1^{\text {st }}$ November 2021 \\ Accepted: $8^{\text {th }}$ December 2021 \\ ${ }^{1}$ Department of Mathematics, Sokoto \\ State University, Sokoto Nigeria. \\ 2Department of Planning, Research and \\ Statistics, National Commission for \\ colleges of Education (NCCE), Abuja, \\ Nigeria. \\ *Corresponding author's email: \\ bangixpat84@gmail.com
}

Cite this: CaJoST, 2022, 1, 10-19

\section{Solving Mixed Boundary Value Problems using Fourier Transform and Dual Integral Equations Method}

\begin{abstract}
Abubakar Umar ${ }^{1 *}$ and Zilkarnaini S. Maishanu²
The research considered the solution of the system of dual integral equations involving Fourier transform occurring in mixed boundary value problems for Laplace's equation with mixed Dirichlet-Neumann boundary conditions. The dual integral equations involving trigonometric function kernel have been solved using Fourier sine transforms. Graphical solutions with the help of Mathematica were used to demonstrate the effectiveness of this method.
\end{abstract}

Keywords: Boundary value problem, Laplace's equation, Mixed boundary value problems, Fourier transform, Dual integral equations.

\section{Introduction}

Dual integral equations are often encountered in different branches of mathematical physics and they generally arise while solving a boundary value problem with mixed boundary conditions [1] and (Debnath et al, 2016). In reducing the corresponding boundary value problem to dual integral equations with a trigonometric function kernel, (Mandal et al, 1994) and (Chakrabarti et al, 1997) studied linear water wave scattering by a vertical barrier. The behaviour of one of the integrals of these dual integral equations at the point where the boundary condition changes play a crucial role in determining their solution. Some dual integral equations involving trigonometric function kernels of the first and second kinds have been studied by [5]. Other types of investigations for the dual integral equations with trigonometric kernels has been done by (Ahdiaghdam et al, 2017), and [7]. This paper focused on the solution of dual integral equations with trigonometric function kernel. However, there are limited contributions to this area especially in the recent years. The paper emphasized on the application of Fourier sine transform to obtained the solution of mixed boundary value problems.

\section{Methodology}

The method used in this paper is Fourier sine transform method to solve Laplace's equation Consider the Fourier sine transform with the given boundary conditions to obtain and solve the dual integral equations given by [8].

\section{Solution of dual integral equations}

Consider Laplace's equation

$$
\frac{\partial^{2} u}{\partial x^{2}}+\frac{\partial^{2} u}{\partial y^{2}}=0, \quad-\infty<x<\infty, \quad 0<y<h,
$$

with the boundary conditions

$$
\begin{aligned}
& \lim _{|x| \rightarrow \infty} u(x, y) \rightarrow 0, \quad 0<y<h_{x} \\
& \left\{\begin{array}{cc}
u\left(x_{x}, 0\right)=\operatorname{sgn}(x), & |x|<1_{x} \\
u_{y}(x, 0)=0, & |x|>1_{x}
\end{array}\right.
\end{aligned}
$$

and

$$
u(x, h)=0, \quad-\infty<x<\infty .
$$

where

$\operatorname{Sgn}(x)= \begin{cases}1_{v} & x>0 \\ 0, & x=0 \\ -1, & x<0,\end{cases}$ 


$$
F_{s}[u(x, y)]=U(\omega, y)=\int_{0}^{\infty} u(x, y) \sin (\omega x) d x .
$$

Taking Fourier sine transform of both sides of Equation (3.1), gives

$$
\begin{aligned}
& F_{g}\left[u_{x x}\right]+F_{s}\left[u_{y y}\right]=0 \\
& \omega u(0, y)+(i \omega)^{2} U+U_{y y}(\omega, y)=0 . \\
& U_{y y}(\omega, y)-\omega^{2} U(\omega, y)=0,
\end{aligned}
$$

Applying the additional condition $u(0, y)=0, \quad 0<y<h$, gives

$$
\frac{\partial^{2} U(\omega, y)}{\partial y^{2}}-\omega^{2} U(\omega, y)=0, \quad 0<y<h,
$$

The characteristic equation is

$$
m^{2}-\omega^{2}=0
$$

which implies

$m= \pm \omega$.

Therefore, the solution has the general form

$$
U(\omega, y)=A(\omega) \sinh (\omega y)+B(\omega) \cosh (\omega y) .
$$

From the boundary condition, we have

$$
u(x, h)=0, \quad-\infty<x<\infty,
$$

$F_{s}[u(x, h)]=U(\omega, h)=0$.

Now Equation (3.7) becomes

$$
\begin{aligned}
& U(\omega, h)=A(\omega) \sinh (\omega h)+B(\omega) \cosh (\omega h)=0, \\
& B(\omega)=-\frac{A(\omega) \sinh (\omega h)}{\cosh (\omega h)} .
\end{aligned}
$$

Substituting $B(\omega)$ in Equation (3.6) gives

$$
\begin{aligned}
U(\omega, y)=A(\omega) \sinh (\omega y) & -\frac{A(\omega) \sinh (\omega h)}{\cosh (\omega h)} \cosh (\omega y) . \\
& =\frac{A(\omega)}{\cosh (\omega h)}[\sinh (\omega y) \cosh (\omega h)-\cosh (\omega h) \sinh (\omega y)] .
\end{aligned}
$$

Applying the identity $\sinh (P-Q)=\sinh P \cosh Q-\cosh P \sinh Q$, we get

$$
U(\omega, y)=\frac{A(\omega) \sinh [\omega(y-h)]}{\cosh (\omega h)} .
$$

Which is equivalent to

$$
U(\omega, y)=\frac{A(\omega) \sinh [\omega(y-h)]}{\cosh (\omega h)},
$$

Taking the inverse Fourier sine transform, we obtain

$$
u(x, y)=\frac{2}{\pi} \int_{0}^{\infty} A(\omega) \frac{\sinh [\omega(y-h)]}{\cosh (\omega h)} \sin (\omega x) d \omega .
$$

Now applying the boundary condition 


$$
u(x, 0)=\operatorname{sgn}(x), \quad|x|<1,
$$

gives

$$
u(x, 0)=\frac{2}{\pi} \int_{0}^{\infty} A(\omega) \frac{\sinh [\omega(0-h)]}{\cosh (\omega h)} \sin (\omega x) \mathrm{d} \omega=\operatorname{sgn}(x) .
$$

By definition of $\operatorname{sgn}(x)$, we get

$$
\frac{2}{\pi} \int_{0}^{\infty} A(\omega) \frac{\sinh (\omega h)}{\cosh (\omega h)} \sin (\omega x) d \omega=-1, \quad 0<x<1,
$$

or

$\frac{2}{\pi} \int_{0}^{\infty} A(\omega) \tanh (\omega h) \sin (\omega x) d \omega=-1, \quad 0<x<1$.

Applying the boundary condition $u_{y}(x, 0)=0, \quad|x|>1_{x}$

$$
u_{y}(x, 0)=\frac{2}{\pi} \int_{0}^{\infty} \omega A(\omega) \frac{\cosh [\omega(0-h)]}{\cosh (\omega h)} \sin (\omega x) d \omega=0,
$$

which simplifies to

$$
\int_{0}^{\infty} \omega A(\omega) \sin (\omega x) d \omega=0, \quad|x|>1 .
$$

Hence, we have the set of dual integral equations (3.10) and (3.11), where $A(\omega)$ is the unknown function.

To obtain the solution of the above dual integral equations, let assume

$$
\omega A(\omega)=\int_{0}^{1} g(t) \sin (\omega t) d t .
$$

Substituting Equation (3.12) into Equation (3.11), we get

$$
\begin{gathered}
\int_{0}^{\infty} \omega A(\omega) \sin (\omega x) d \omega=-\frac{d}{d x}\left[\int_{0}^{\infty} A(\omega) \cos (\omega x) d \omega\right] \\
\quad=-\int_{0}^{1} g(t) \frac{d}{d x}\left[\int_{0}^{\infty} \sin (\omega t) \cos (\omega x) \frac{d \omega}{\omega}\right] d t \\
=0 .
\end{gathered}
$$

Applying (Gradshteyn and Ryzhik, 1980, (3.741.2), p 414)) The integral within the square bracket varnishes since $|x|>1$ and $0 \leq t \leq 1$. Thus Equation (3.12) satisfies Equation (3.11).

To obtain $g(t)$, substitute Equation (3.12) into Equation (3.10)

$$
\int_{0}^{1} g(t)\left[\int_{0}^{\infty} \tanh (\omega h) \sin (\omega x) \sin (\omega t) \frac{d \omega}{\omega}\right] d t=-\frac{\pi}{2} .
$$

The integral within the square bracket, denoted by I, in the above equation can be evaluated as follows:

Using $\sin p \sin q=\frac{1}{2}(\cos (p-q)-\cos (p+q))$ with $p=\omega x, q=\omega t$. 
we obtain

$$
I=\int_{0}^{\infty} \tanh (\omega h)\left[\frac{1}{2}(\cos \omega(x-t)-\cos \omega(x+t))\right] \frac{d \omega}{\omega} .
$$

Applying the formula from (Gradshteyn \& Ryzhik, 1980, (1965, (4.116.2), p 516))

$$
\int_{0}^{\infty} \cos (\omega \omega) \tanh (\beta \omega) \frac{d \omega}{\omega}=\ln \left|\operatorname{coth} \frac{\alpha \pi}{4 \beta}\right| .
$$

we obtained

$$
\begin{aligned}
\mathrm{I} & =\frac{1}{2} \ln \left|\operatorname{coth} \frac{(x-t) \pi}{4 h}\right|-\frac{1}{2} \ln \left|\operatorname{coth} \frac{(x+t) \pi}{4 h}\right| \\
& =\frac{1}{2}[\ln |\operatorname{coth}(\phi(x-t))|-\ln |\operatorname{coth}(\phi(x+t))|],
\end{aligned}
$$

where $\varphi=\frac{\pi}{4 h}$.

Applying the property of logarithms, we get

$$
\begin{aligned}
I & =\frac{1}{2} \ln \left|\frac{\operatorname{coth}(\varphi(x-t))}{\operatorname{coth}(\varphi(x+t))}\right| \\
& =\frac{1}{2} \ln \left|\frac{\operatorname{coth}(\varphi x-\varphi t)}{\operatorname{coth}(\varphi x+\varphi t)}\right| \\
& =\frac{1}{2} \ln \left|\frac{\tanh (\varphi x+\varphi t)}{\tanh (\varphi x-\varphi t)}\right| \\
& =\frac{1}{2} \ln \left|\frac{\frac{\sinh (\varphi x+\varphi t)}{\sinh (\varphi x+\varphi t)}}{\cosh (\varphi x-\varphi t)}\right| \\
& =\frac{1}{2} \ln \left|\frac{\sinh \varphi(x+t)}{\cosh \varphi(x+t)} \frac{\cosh \varphi(x-t)}{\sinh \varphi(x-t)}\right|
\end{aligned}
$$

Applying the identity

$$
\sinh X \pm \sinh Y=2 \sinh \frac{1}{2}(X \pm Y) \frac{1}{2} \cosh (X \mp Y)
$$

which is equivalent to

$$
\sinh (X \pm Y) \cosh (X \mp Y)=\frac{1}{2}[\sinh (2 X) \pm \sinh (2 Y)]
$$

we obtained

$$
\begin{aligned}
I=\frac{1}{2} \ln \left|\frac{\sinh \varphi(x+t) \cosh \varphi(x-t)}{\cosh \varphi(x+t) \sinh \varphi(x-t)}\right| & =\frac{1}{2} \ln \left|\frac{\sinh 2 \varphi x+\sinh 2 \varphi t}{\sinh 2 \varphi x-\sinh 2 \varphi t}\right| x \\
= & \frac{1}{2} \ln \left|\frac{\sinh [\pi x /(2 h)]+\sinh [\pi t /(2 h)]}{\sinh [\pi x /(2 h)]-\sinh [\pi t /(2 h)]}\right| .
\end{aligned}
$$

and Equation (3.14) becomes 


$$
\int_{0}^{1} g(t) \ln \left|\frac{\sinh [\pi t /(2 h)]+\sinh [\pi x /(2 h)]}{\sinh [\pi t /(2 h)]-\sinh [\pi x /(2 h)]}\right| d t=-\pi .
$$

Let

$$
\xi=\frac{\sinh [\pi t /(2 h)]}{\sinh [\pi /(2 h)]}, \quad \eta=\frac{\sinh [\pi x /(2 h)]}{\sinh [\pi /(2 h)]} .
$$

Then

$$
t=\frac{2 h}{\pi} \operatorname{arcsinh}\left[\xi \sinh \left(\frac{\pi}{2 h}\right)\right]
$$

$$
\begin{aligned}
\frac{d t}{d \xi} & =\frac{2 h}{\pi} \frac{d}{d \xi}\left\{\sinh ^{-1}\left[\xi \sinh \left(\frac{\pi}{2 h}\right)\right]\right\} \\
& =\frac{2 h}{\pi} \frac{1}{\sqrt{\left[\xi \sinh \left(\frac{\pi}{2 h}\right)\right]^{2}+1}} \frac{d}{d \xi}\left[\xi \sinh \left(\frac{\pi}{2 h}\right)\right]
\end{aligned}
$$

Hence

$$
d t=\frac{2 h \sinh \left(\frac{\pi}{2 h}\right)}{\pi \sqrt{\xi^{2} \sinh ^{2}\left(\frac{\pi}{2 h}\right)+1}} d \xi
$$

and

$g(t)=g\left\{\frac{2 h}{\pi} \operatorname{arcsinh}\left[\xi \sinh \left(\frac{\pi}{2 h}\right)\right]\right\}$.

This implies

$$
F(\xi)=g\left\{\frac{2 h}{\pi} \operatorname{arcsinh}\left[\xi \sinh \left(\frac{\pi}{2 h}\right)\right]\right\} \frac{\sinh [\pi /(2 h)]}{{\left.\sqrt{1+\xi^{2} \sinh ^{2}[\pi /(2 h)}\right]}^{x}}
$$

Equation (3.45) becomes

$$
\int_{0}^{1} g\left\{\frac{2 h}{\pi} \operatorname{arcsinh}\left[\xi \sinh \left(\frac{\pi}{2 h}\right)\right]\right\} \frac{\sinh [\pi /(2 h)]}{\sqrt{1+\xi^{2} \sinh ^{2}[\pi /(2 h)]}} \ln \left|\frac{\xi+\eta}{\xi-\eta}\right| d \xi=-\frac{\pi^{2}}{2 h}(3.16)
$$

Equation (3.16) can be written as

$$
\int_{0}^{1} F(\xi) \ln \left|\frac{\xi+\eta}{\xi-\eta}\right| d \xi=-\frac{\pi^{2}}{2 h} \text {. }
$$

According to [10] the solution of the integral equation

$$
\int_{0}^{1} \ln \left|\frac{x+t}{x-t}\right| h(t) d t=\pi f(x), \quad 0<x<1
$$


is given by

$h(t)=-\frac{2}{\pi} \frac{d}{d t}\left[\int_{t}^{1} \frac{\alpha s(\alpha)}{\sqrt{\alpha^{2}-t^{2}}} d \alpha\right]+\frac{2 f\left(0^{+}\right)}{\pi t \sqrt{1-t^{2}}}$.

where

$s(\alpha)=\int_{0}^{\infty} \frac{f^{\prime}(\xi)}{\sqrt{\alpha^{2}-\xi^{2}}} d \xi$

Equating the right-hand-side of Equations (3.17) and (3.18) we obtain

$\pi f(x)=-\frac{\pi^{2}}{2 h}$

$f(x)=-\frac{\pi}{2 h}$

Now, by replacing $x=\eta$, and $t=\xi$, in Equation (3.18) we have

$$
\int_{0}^{1} F(\xi) \ln \left|\frac{\xi+\eta}{\xi-\eta}\right| d \xi=f(\eta)
$$

$F(\xi)=-\frac{2}{\pi} \frac{d}{d \xi}\left[\int_{\xi}^{1} \frac{\omega s(\alpha)}{\sqrt{\alpha^{2}-\xi^{2}}} d \alpha\right]+\frac{2 f\left(0^{+}\right)}{\pi \xi \sqrt{1-\xi^{2}}}$

Where

$s(\alpha)=\int_{0}^{\infty} \frac{f^{\prime}(u)}{\sqrt{\alpha^{2}-u^{2}}} d u$.

This implies that,

Therefore,

$$
f(\eta)=-\frac{\pi}{2 h} \Rightarrow f^{\prime}(\eta)=0 \Rightarrow s(\alpha)=0,
$$

Substituting,

$$
f\left(0^{+}\right)=-\frac{\pi}{2 h}
$$

Then we have,

$$
F(\xi)=\frac{2\left(-\frac{\pi}{2 h}\right)}{\pi \xi \sqrt{1-\xi^{2}}}=-\frac{1}{h \xi \sqrt{1-\xi^{2}}}
$$

$$
-\frac{1}{h \xi \sqrt{1-\xi^{2}}}=\frac{g(t) \sinh [\pi /(2 h)]}{\sqrt{1+\xi^{2} \sinh ^{2}[\pi /(2 h)]}}
$$

which implies

$$
g(t)=-\frac{\sqrt{1+\xi^{2} \sinh ^{2}[\pi /(2 h)]}}{\sinh [\pi /(2 h)] h \xi \sqrt{1-\xi^{2}}} \times
$$

Now, substituting $\xi=\frac{\sinh [\pi t /(2 h)]}{\sinh \left[\pi_{(2 h)}\right]}$ we have

$$
g(t)=-\frac{\sqrt{1+\frac{\sinh ^{2}[\pi t /(2 h)]}{\sinh ^{2}[\pi /[2 h)]} \sinh ^{2}[\pi /(2 h)]}}{h \sinh [\pi /(2 h)] \frac{\sinh [\pi t /(2 h)]}{\sinh [\pi /(2 h)]} \sqrt{1-\frac{\sinh ^{2}[\pi t /(2 h)]}{\sinh ^{2}[\pi /(2 h)]}}}
$$

After some cancellations we have 


$$
\begin{gathered}
g(t)=-\frac{\sqrt{1+\sinh ^{2}[\pi t /(2 h)]}}{h \sinh [\pi t /(2 h)] \sqrt{1-\frac{\sinh ^{2}[\pi t /(2 h)]}{\sinh ^{2}[\pi /[(2 h)]}}} \\
=-\frac{\sqrt{\cosh ^{2}[\pi t /(2 h)]}}{h \sinh [\pi t /(2 h)] \frac{\sqrt{\sinh ^{2}[\pi /[2 h)]-\sinh ^{2}[\pi t /[2 h)]}}{\sinh [\pi /(2 h)]}}
\end{gathered}
$$

On simplifications we obtained

$$
\begin{aligned}
g(t)= & -\frac{\cosh [\pi t /(2 h)] \sinh [\pi /(2 h)]}{h \sinh [\pi t /(2 h)]} \frac{1}{\sqrt{\sinh ^{2}[\pi /(2 h)]-\sinh ^{2}[\pi t /(2 h)]}} \\
& =-\frac{\cosh [\pi t /(2 h)] \sinh [\pi /(2 h)]}{1 / \sqrt{2} h \sqrt{\cosh (\pi t / h)-1}} \frac{1}{\sqrt{\frac{\cosh (\pi / h)-1}{2}-\frac{\cosh (\pi t / h)-1}{2}}} \\
= & -\frac{\cosh [\pi t /(2 h)] \sinh [\pi /(2 h)]}{1 / \sqrt{2} h \sqrt{\cosh (\pi t / h)-1}} \frac{1}{1 / \sqrt{2} \sqrt{\cosh (\pi / h)-\cosh (\pi t / h)}} \\
= & -\frac{\cosh [\pi t /(2 h)] \sinh [\pi /(2 h)]}{1 / 2 h \sqrt{\cosh (\pi t / h)-1} \sqrt{\cosh (\pi / h)-\cosh (\pi t / h)}}
\end{aligned}
$$

Therefore,

$$
g(\mathrm{t})=-\frac{2 \cosh [\pi t /(2 h)] \sinh [\pi /(2 h)]}{h \sqrt{\cosh (\pi t / h)-1} \sqrt{\cosh (\pi / h)-\cosh (\pi t / h)}} .
$$

Similarly, by replacing Equation (3.19) into Equation (3.12) and then substituting the result into Equation (3.19), the completed integral solution of the given boundary value problem is obtained.

\section{Results and Discussion}

The result of the problem $u(x, y)$ can be obtain by combining equation (3.19) and Equation (3.9) as

$$
u(x, y)=\frac{2}{\pi} \int_{0}^{\infty}\left[\int_{0}^{1} \frac{g(t) \sin (\omega t) \sinh [\omega(y-h)]}{\omega \cosh (\omega h)} \sin (\omega x) d t\right] d \omega .
$$

To plot the graphs of $u(x, y)$, we simplify the above equation as follows:

Let

$$
\begin{aligned}
r(t)=\int_{0}^{\infty} \frac{\sin (\omega t) \sinh [\omega(y-h)] \sin (\omega x)}{\omega \cosh (\omega h)} d \omega, & \\
& =\int_{0}^{\infty} \frac{\sin (\omega t) \sinh (\omega A) \sin (\omega x)}{\omega \cosh (\omega h)} d \omega,
\end{aligned}
$$

where $A=y-h$

$$
r(t)=\int_{0}^{\infty} \sin (\omega t) \sin (\omega x) \frac{\sinh (\omega A)}{\omega \cosh (\omega h)} d \omega,
$$

Using the identity that, 


$$
\sin A \sin B=\frac{1}{2}[\cos (A-B)-\cos (A+B)]
$$

we have

$$
\begin{aligned}
\sin (\omega t) \sin (\omega x) & =\frac{1}{2}[\cos (\omega t-\omega x)-\cos (\omega t+\omega x)]_{x} \\
& =\frac{1}{2}[\cos (t-x) \omega-\cos (t+x) \omega] .
\end{aligned}
$$

Substituting, we attained

$$
\begin{aligned}
r(t)=\frac{1}{2}\left[\int_{0}^{\infty} \cos (t-x) \omega-\cos (t+x) \omega \frac{\sinh (\omega A)}{\omega \cosh (\omega h)} d \omega\right] & \\
= & \frac{1}{2}\left[\int_{0}^{\infty} \frac{\cos (t-x) \omega \sinh (\omega A)}{\omega \cosh (\omega h)} d \omega-\int_{0}^{\left.\int_{0}^{\infty} \frac{\cos (t+x) \omega \sinh (\omega A)}{\omega \cosh (\omega h)} d \omega \cdot\right]}\right. \\
r_{1}(t) & r_{2}(t)
\end{aligned}
$$

This implies

$$
r(t)=\frac{r_{1}(t)-r_{2}(t)}{2} .
$$

Using the relation from (Gradshteyn \& Ryzhik, 1980,(4.114.2), p 513) )

$$
\int_{0}^{\infty} \frac{\cos (\alpha \omega)}{\omega} \frac{\sinh (\beta \omega)}{\cosh (\gamma \omega)} d \omega=\frac{1}{2} \ln \left[\frac{\cosh \left(\frac{\alpha \pi}{2 \gamma}\right)+\sin \left(\frac{\beta \pi}{2 \gamma}\right)}{\cosh \left(\frac{\alpha \pi}{2 \gamma}\right)-\sin \left(\frac{\beta \pi}{2 \gamma}\right)}\right] x
$$

For $r_{1}(t)$ apply the above formula with $a=t-x_{s} \beta=A_{s} y=h$ we get

$r_{1}(t)=\frac{1}{2} \ln \left[\frac{\cosh \left(\frac{(t-x) \pi}{2 h}\right)+\sin \left(\frac{A \pi}{2 h}\right)}{\cosh \left(\frac{(t-x) \pi}{2 h}\right)-\sin \left(\frac{A \pi}{2 h}\right)}\right]$.

For $r_{2}(t)$ apply the above formula with $a=t+x_{s} \beta=A_{s} y=h$. we get

$$
r_{2}(t)=\frac{1}{2} \ln \left[\frac{\cosh \left(\frac{(t+x) \pi}{2 h}\right)+\sin \left(\frac{A \pi}{2 h}\right)}{\cosh \left(\frac{(t+x) \pi}{2 h}\right)-\sin \left(\frac{A \pi}{2 h}\right)}\right] .
$$

Therefore,

$$
\begin{gathered}
r(t)=\frac{1}{4}\left\{\ln \left[\frac{\cosh \left(\frac{(t-x) \pi}{2 h}\right)+\sin \left(\frac{A \pi}{2 h}\right)}{\cosh \left(\frac{(t-x) \pi}{2 h}\right)-\sin \left(\frac{A \pi}{2 h}\right)}\right]-\ln \left[\frac{\cosh \left(\frac{(t+x) \pi}{2 h}\right)+\sin \left(\frac{A \pi}{2 h}\right)}{\cosh \left(\frac{(t+x) \pi}{2 h}\right)-\sin \left(\frac{A \pi}{2 h}\right)}\right]\right\} x \\
=\frac{1}{4} \ln \left[\frac{\cosh \left(\frac{(t-x) \pi}{2 h}\right)+\sin \left(\frac{A \pi}{2 h}\right)}{\cosh \left(\frac{(t-x) \pi}{2 h}\right)-\sin \left(\frac{A \pi}{2 h}\right)} \frac{\cosh \left(\frac{(t+x) \pi}{2 h}\right)-\sin \left(\frac{A \pi}{2 h}\right)}{\cosh \left(\frac{(t+x) \pi}{2 h}\right)+\sin \left(\frac{A \pi}{2 h}\right)}\right] .
\end{gathered}
$$

where

$$
A=y-h
$$

$$
r_{1}(t)=\cosh \left(\frac{(t-x) \pi}{2 h}\right)+\sin \left(\frac{A \pi}{2 h}\right)
$$




$$
\begin{aligned}
& r_{2}(t)=\cosh \left(\frac{(t+x) \pi}{2 h}\right)-\sin \left(\frac{A \pi}{2 h}\right) \\
& r_{3}(t)=\cosh \left(\frac{(t-x) \pi}{2 h}\right)-\sin \left(\frac{A \pi}{2 h}\right) \\
& r_{4}(t)=\cosh \left(\frac{(t+x) \pi}{2 h}\right)+\sin \left(\frac{A \pi}{2 h}\right) .
\end{aligned}
$$

Substituting back, Equation (3.9) becomes

$$
u(x, y)=\frac{1}{2 \pi} \int_{0}^{1} g(t) r(t) d t .
$$

we plotted the graph of the solution $u(x, y)$

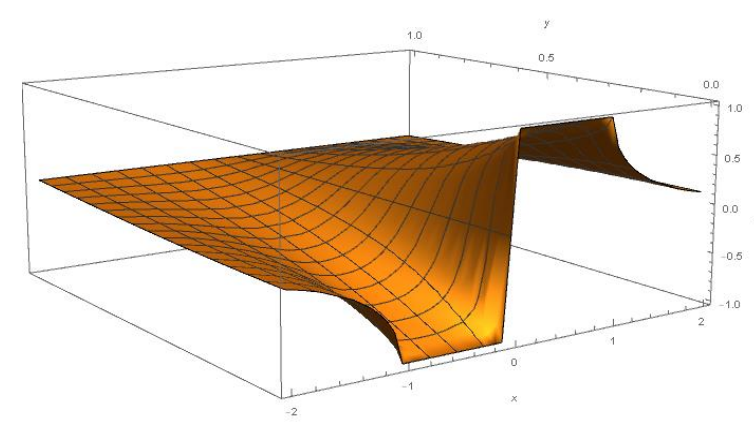

Figure 1. The graph of the solution $u(x, y)$ with $h=1$

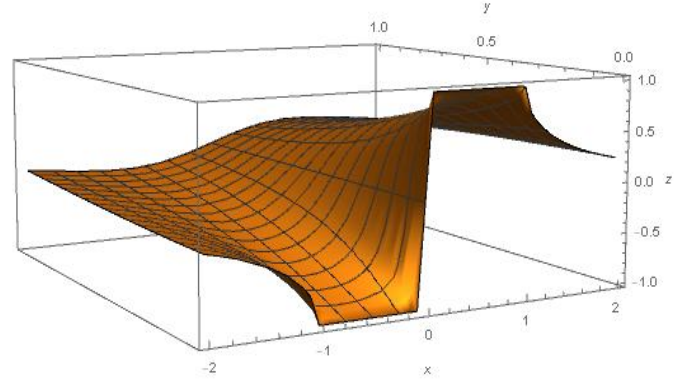

Figure 2. The graph of the solution $u(x, y)$ with $h=2$

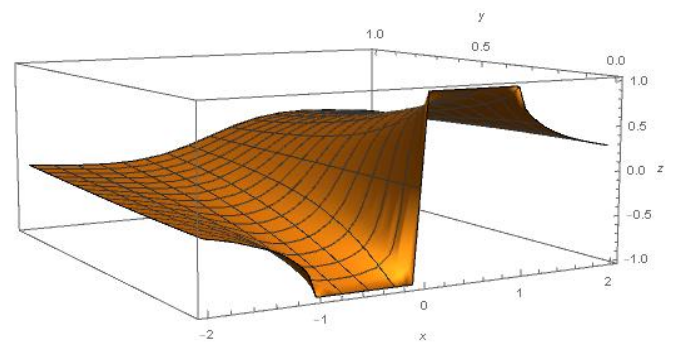

Figure 3. The graph of the solution $u(x, y)$ with $h=3$

\section{Conclusion}

In this paper, we have discussed the various results generated in 3D graphs using different values of $h$, it is observed in Figure 2 and Figure 3 that the graph generated when $h=2$ and $h=3$ gives the same result but not as accurate as when $h=1$. However, the result obtained in Figure 1 shows that the graph generated in 3D remains unchanged since $k$ is well defined.

\section{Acknowledgements}

The authors thank Dr. M. Ibrahim and Dr. S. Shehu for some useful discussions. This work is partially supported by Department of Mathematics, Sokoto State University, Sokoto.

\section{Conflict of interest}

The authors declare no conflict of interest.

\section{References}

[1] I. N. Sneddon, "The Use of Integral Transforms McGraw-Hill," New York, pp. 235-238, 1972.

[2]

L. Debnath and D. Bhatta, Integral transforms and their applications. Chapman and Hall/CRC, 2016.

[3] C. and N. Mandal, "Solution of some Dual integral equations," 1994.

[4] A. Chakrabarti, S. Banerjea, B. N. Mandal, and T. Sahoo, "SURFACE WATER WAVES BY VERTICAL BARRIERS," vol. 39, no. October 1994, pp. 93-103, 1997.

[5] C. Nasim, "On dual integral equations with Hankel kernel and an arbitrary weight function," Int. J. Math. Math. Sci., vol. 9, 1986. 
[6] S. Ahdiaghdam, S. Shahmorad, and K. Ivaz, "Approximate solution of dual integral equations using Chebyshev polynomials," Int. J. Comput. Math., vol. 94, no. 3, pp. 493-502, 2017, doi: 10.1080/00207160.2015.1114611.

[7] S. K. Banerjea, "A Note on some Dual Integral Equations," 2000.

[8] D. G. Duffy, Mixed boundary value problems. CRC Press, 2008.

[9] I. S. Gradshteyn and I. M. Ryzhik, "Tables of Integrals, Series, and Products Academic Press," Inc., San Diego, 1980.

[10] J. C. Cooke, "The solution of some integral equations and their connection with dual integral equations and series," Glas. Math. J., vol. 11, no. 1, pp. 9-20, 1970. 